\title{
ATROFIA PROGRESSIVA DE RETINA EM UM GATO SEM RAÇA DEFINIDA, TENDO COMO PRINCIPAL DIAGNÓSTICO DIFERENCIAL A RETINOPATIA TÓXICA - RELATO DE CASO
}

(Progressive retinal atrophy in a mongrel cat, being the toxic retinopathy the main differential diagnosis - case report)

\section{Manuella Oloveira Borges de Sampaio, Nathália Mendonça de Seabra, Fabiano Montiani-Ferreira ${ }^{1}$}

\section{${ }^{1}$ Correspondência: montiani@ufpr.br}

RESUMO: Atrofia progressiva de retina (APR) é o termo utilizado para generalizar uma variedade de condições hereditárias que causam distrofia ou degeneração retiniana em animais. Em gatos, doenças oculares hereditárias, tais como a APR, são incomuns quando comparadas às suas frequências em cães. $O$ caso a ser relatado é de uma gata sem raça definida, 11 anos, que apresentou sinais clínicos muito semelhantes aos de Atrofia Progressiva de Retina. A paciente apresentava, também, histórico pregresso de tratamento intenso com antibioticoterapia no seu primeiro ano de vida. A Retinopatia Tóxica induzida pelo uso indiscriminado de enrofloxacina em gatos é um dos diagnósticos diferenciais de APR. O caso relatado se torna atípico por se tratar de uma gata sem raça definida, já que a Atrofia Progressiva de Retina é uma doença que, quando acomete gatos, fato este mais raro que em cães, geralmente acomete a raça Abssínio, Somali, Siamês e Persa. Apesar de o histórico da paciente não condizer com as características de uma retinopatia tóxica, não há como excluir completamente essa possibilidade, por mais que a droga tenha sido administrada há 10 anos, pois nos estágios finais da APR as alterações retinianas são semelhantes às de intoxicação por enrofloxacina. Para a confirmação definitiva do diagnóstico, seria necessário realizar um teste genético para a identificação do alelo responsável pela doença.

Palavras-chave: gato, atrofia progressiva de retina, degeneração retiniana, retinopatia tóxica

ABSTRACT:. Progressive Retinal Atrophy (PRA) is the term used to generalize a variety of conditions that cause inherited retinal dystrophy or retinal degeneration in animals. In cats, hereditary eye diseases such as PRA are uncommon compared to their frequencies in dogs. The case to be reported is a mongrel cat, 11, who showed clinical signs very similar to Progressive Retinal Atrophy. The patient had also past history of intense treatment with antibiotics in the first year of life. The Toxic retinopathy induced by the use indiscriminate of enrofloxacin in cats is one differential diagnoses for PRA. The reported case becomes atypical because the Retina Atrophy Progressive is a disease that, when it affects cats, a fact rarer than in dogs, usually affects the Abssínio breed, Somali, Siamese and Persian. In this case, the patient is a mongrel cat. Although the patient's history isn't compatible with toxic retinopathy, this possibility isn't ruled out completely, even if the drug was given 10 years ago, because the final stages of the PRA the retinal changes are similar to intoxication for enrofloxacin. So, for the final confirmation of the diagnosis would be necessary to carry out a genetic test for identification of the allele responsible for the disease.

Key Words: cat, progressive retinal atrophy, retinal degeneration, toxic retinopathy 


\section{INTRODUÇÃO}

Atrofia progressiva de retina (APR) é o termo utilizado para generalizar uma variedade de condições hereditárias que causam distrofia ou degeneração retiniana em animais. Tais condições geralmente envolvem uma degeneração progressiva dos fotorreceptores da retina, com variações na idade de início e progressão da doença (PETERSEN-JONES, 1998). A APR é, dentre as degenerações retinianas hereditárias, a mais frequente em cães (PETERSEN-JONES, 2005). Já foram relatadas formas de APR em gatos da raça Siamês, gatos domésticos de pelo curto (GIULIANO \& VAN DER WOERDT, 1999) no Persa (RUBIN \& LIPTON, 1973) Abissínio e Somali (MENOTTI-RAYMOND et al.2007). No entanto, em gatos, doenças oculares hereditárias são incomuns quando comparadas às suas frequências em cães (GLAZE, 2005).

A primeira degeneração de retina felina molecularmente caracterizada foi a que ocorre na raça Abissínio e pode ser comparada à Amaurose Congênita de Leber, um tipo de APR (MENOTTIRAYMOND et al.2007) ou retinose pigmentar, que é a forma que a oftalmologia médica se refere à APR. Foi descrita na Suécia por Narfström, K. (1983), apresentando caráter recessivo do alelo denominado "rdAc" (degeneração retiniana do gato Abissínio).

O alelo rdAc tem uma distribuição mundial em Abissínios e em gatos da raça Somali, com impacto clínico significativo em gatos homozigotos afetados. Em decorrência disso, um estudo realizado por Menotti-raymond et al.(2010) buscou determinar se o alelo rdAc está presente em outras raças. Neste estudo, 43 novas raças, totalizando 846 indivíduos, foram genotipados para 0 alelo rdAc. Surpreendentemente, o alelo $\mathrm{rdAc}$ foi detectado em $34 \%$ dos gatos distribuídos entre as diversas raças e com frequências relativamente altas em Siameses e raças próximas aos Siameses, tanto da América do Norte quanto da Europa. Do total de indivíduos detectados para o alelo rdAc, 27 gatos apresentaram homozigose e evidências clínicas de degeneração da retina.

Diferentemente do que foi verificado nos gatos da raça Abssínio que manifestam a doença de forma tardia, Rah et al.(2005) observou em filhotes de gatos da raça Persa nascidos de pais com APR, sinais oftálmicos precoces e progressivos de degeneração de retina. De acordo com o autor, a atenuação dos vasos da retina iniciou-se na quarta semana de vida, sendo que entre 16 e 17 semanas de idade, os vasos já eram vistos com dificuldade. Além disso, o autor relata início do afinamento da retina com nove semanas por meio da presença de focos de hiperrefletividade, a qual se generalizou entre 16 e 17 semanas. Outro estudo realizado por Giuliano \& Van der Woerdt (1999) descreveu a forma hereditária de APR na raça Siamês. Assim, como descrito no Abssínio em 1983, em ambos os estudos a doença apresentou hereditariedade de caráter recessivo.

Apesar de a doença apresentar predominância de hereditariedade de caráter recessivo nos estudos descritos na literatura, Curtis e Barnett (1985) e Curtis et. al (1987) descreveram em gatos Abissínios outra forma de APR, a qual revelou herança autossômica dominante e características diferentes no desenvolvimento da doença. Os primeiros sinais foram de início precoce, entre 8 e 12 semanas de vida, caracterizando uma displasia de cones e bastones, diferente da degeneração de retina caracterizada pela herença recessiva anteriormente descrita nesta raça. O símbolo adotado para o gene foi "Rdy". 
De tudo o que já se sabe sobre a APR nos animais e nos seres humanos, pode-se afirmar que se trata de uma doença degenerativa de caráter bilateral e tende à simetria (GELATT 2003). Não há predisposição sexual para sua ocorrência (BEDFORD, 2005) e os pacientes afetados apresentam, inicialmente, nictalopia (cegueira noturna), uma vez que os bastonetes são acometidos primeiro (NARFSTRÖM et al.,2011). Neste momento, observa-se apenas a perda da visão periférica (SANDE, 2010). Com o tempo se determina também acromatopsia (cegueira diurna), devido à morte dos cones (NARFSTRÖM et al.,2011), a qual progride da periferia ao centro do campo de visão, até que ocorra perda completa da visão (SANDE, 2010).

O diagnóstico se baseia no histórico completo e no exame oftalmológico minucioso. Neste, são avaliados reflexos pupilares diretos e consensuais, reflexo de ofuscamento, e teste de ameaça (HERRERA, 2007). O melhor instrumento diagnóstico para a detecção clínica precoce da APR é a eletrorretinografia (ERG), que pode ser considerada como teste "Padrão Ouro". Em relação ao reflexo pupilar direto e consensual, sua presença não implica em uma retina com função normal; mesmo estando presentes, ela pode estar degenerada (GROZDANIC et al.,2006). Portanto, o reflexo de ofuscamento, manifestado pelo piscar de olhos na presença de luz forte, é considerado um melhor avaliador da função retiniana, pois, quando existe lesão retiniana grave, lesão do disco óptico ou do nervo facial, esse reflexo está ausente (GOMES et al.,2013). O teste de ameaça também avalia a função normal da retina e consiste em fazer um movimento próximo aos olhos do paciente a fim de que haja a resposta voluntária do piscar de olhos ou movimento de cabeça (HERRERA, 2007).
O segmento posterior do olho e mais especificamente a retina são examinados em uma sala escura com auxílio de oftalmoscopia indireta ou direta, podendo ser necessário o auxílio da ultrassonografia ocular e da eletrorretinografia.

Durante a oftalmoscopia, deve-se examinar os vasos retinianos, avaliando o número, distribuição e calibre dos mesmos. Se os vasos coroidais forem visíveis, estes também devem ser observados. A área tapetal é avaliada quanto à refletividade, se está atenuada ou exacerbada (GELATT, 2003). Na APR, observase hiperrefletividade da área tapetal pelo afinamento da retina e atenuação dos vasos sanguíneos primeiramente na retina periférica, seguida da alteração no centro da retina (SANDE, 2010). Diferentemente do que ocorre em cães, nos gatos não se observa um aglomerado de pigmentação na região não tapetal, bem como palidez do disco óptico ou catarata (NARFSTRÖM, K et al., 1988).

Como já mencionado, para confirmar o diagnóstico de APR, realizase a eletrorretinografia (SANDE, 2010), por meio da qual se determina disfunção e morte celular na retina. $A$ eletrorretinografia (ERG) revela diminuição progressiva da amplitude pico a pico (pico da onda-a ao pico da ondab) e aumento do tempo de culminação da onda-b, representada pela onda registrada desde o início do estímulo luminoso até o pico máximo dessa onda (NARFSTRÖM \& EKESTEN, 1998). Pode-se, também haver ausência de respostas, resultando em ERG extinto (SAFATLE et al.,2010). A fase escotópica do exame é mais precocemente afetada devido à perda rápida da função dos bastonetes (NARFSTRÖM \& EKESTEN, 1998).

O diagnóstico diferencial é importante para descartar outras causas de diminuição da capacidade visual. Para determinação deste diagnóstico, 
avalia-se primeiramente o histórico do paciente, $o$ início dos sintomas e a evolução da doença. Além disso, devemse considerar os resultados observados durante o exame oftalmológico clínico, os exames complementares, como o ERG e o ultrassom ocular (SLATTER, 2005).

Por meio deste conjunto, é possível identificar se a diminuição visual está relacionada a uma alteração anatômica, como um descolamento retiniano ou uma possível degeneração da retina (SLATTER, 2005) ou a outra patologia ocular.

O caso a ser relatado é de uma gata sem raça definida, 11 anos, que apresentou sinais muito semelhantes aos de Atrofia Progressiva de Retina, apesar de apresentar um histórico pregresso de tratamento intenso com antibioticoterapia 10 anos antes do aparecimento de tais sinais. É importante considerar essa informação uma vez que a Retinopatia Tóxica caracterizada pelo uso indiscriminado de enrofloxacina em gatos é um dos diagnósticos diferenciais.

\section{Relato do Caso}

Em maio de 2014, uma gata de 11 anos, sem raça definida (Figura 1), foi atendida pelo Serviço de Oftalmologia do Hospital Veterinário localizado na cidade de Curitiba - Paraná. Segundo o proprietário, a paciente começou a apresentar sinais de que não estava enxergando bem nos últimos meses, que se intensificaram progressivamente no período que antecedeu a consulta. De acordo com o proprietário, a gata era muito esperta e, mesmo com a dificuldade visual, aprendeu rapidamente a se deslocar, mesmo em locais desconhecidos e por isso já não a observavam se chocar com frequência contra os móveis em casa. Contudo, foi relatado que a gata não localizava facilmente as pessoas em casa, movimentando-se com bastante cautela, a fim de sentir qualquer movimentação por meio de suas vibrissas (bigodes).

Segundo o histórico apresentado pelo proprietário, a paciente sempre se alimentou com ração para gatos da marca Hills®, não possuía acesso à rua, estava com todas as vacinas em dia, a vermifugação era feita regularmente e possuía contato com outros cães e gatos que viviam na mesma casa. Todos os contactantes eram saudáveis, vacinados, vermifugados e sem sinais de déficit visual, bem como qualquer outra alteração. O proprietário não tinha informações sobre o histórico dos parentes da gata, no que diz respeito a alterações oftálmicas e possíveis anormalidades hereditárias.

Além disso, o proprietário referiu que, com um ano de idade, a paciente foi atacada por um cachorro e ficou internada durante aproximadamente um mês em uma clínica veterinária, onde foi realizada a administração de uma série de medicamentos, dentre eles, antibióticos, mas não soube dizer quais.

Por meio do exame clínico e inspeção minuciosa dos olhos, os quais serão relatados posteriormente, a paciente foi diagnosticada com Atrofia Progressiva de Retina. Em agosto de 2014, ela retornou ao Hospital Veterinário para acompanhamento da evolução, progressão da doença e realização da eletrorretinografia.

Figura 1 - Foto da paciente no Setor de Oftalmologia - Hospital Veterinário.

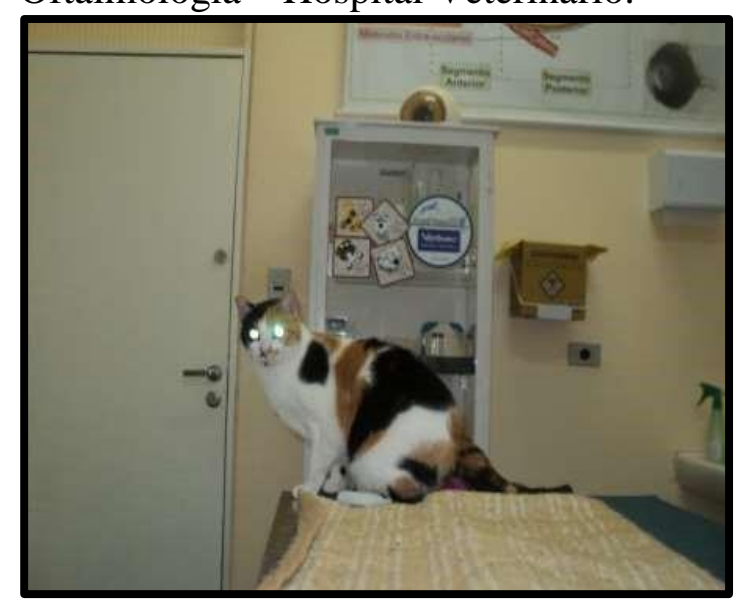


Durante o exame clínico em maio de 2014, observou-se ausência de reflexo pupilar direto, de reflexo pupilar consensual e de resposta à ameaça em ambos os olhos. A paciente não apresentou reflexo de ofuscamento em resposta à fonte de luz, já o reflexo palpebral se apresentou normal. Não se observaram alterações nas pálpebras e cílios, assim como em aparelho lacrimal. Notou-se uma opacidade corneana pontual, chamada Florida Spots na região de 2 horas na córnea do olho esquerdo (Figura 2), alteração essa idiopática e mais comum em gatos que vivem em clima tropical. O teste com o corante de fluoresceína foi negativo em ambos os olhos e não foram observadas alterações em conjuntiva palpebral, conjuntiva bulbar e $3^{a}$ pálpebra. No segmento anterior do olho, a úvea anterior, humor aquoso, câmara anterior e ângulo de drenagem se apresentavam normais, havia apenas midríase bilateral (Figura 3). A lente não apresentava opacidade, bem como vestígios de luxação e o humor vítreo não exibia alterações.

Figura 2 - Foto da paciente durante exame clínico em consulta Setor de Oftalmologia do Hospital Veterinário em agosto de 2014. No olho esquerdo, observase a lesão tipo Florida spots, indicada pela seta.

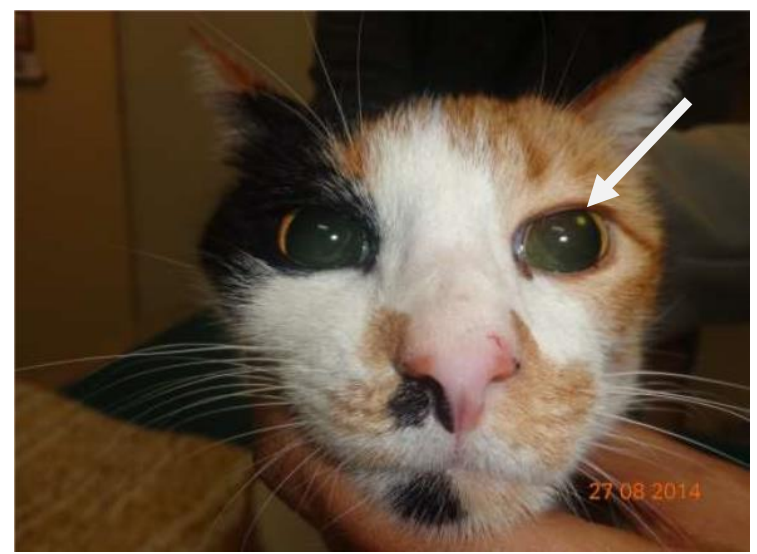

Figura 3 - Foto da paciente durante exame clínico em consulta no Setor de Oftalmologia do Hospital Veterinário em agosto de 2014. Notar a midríase bilateral e ausência de outras alterações no segmento anterior dos olhos. A: Olho esquerdo. B: Olho direito.

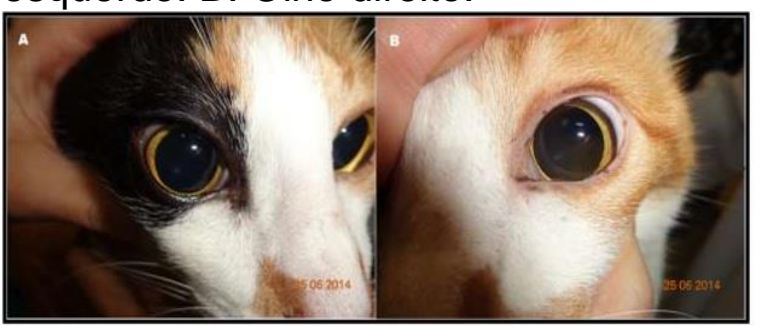

Ao avaliar a retina, observou-se ausência da vascularização local e focos de hiperrefletividade simétricos em região tapetal em ambos os olhos. Não havia sinais de coriorretinite ou alterações no disco óptico.

Durante a consulta, foi realizado o teste de obstáculos, com a iluminação da sala presente e ausente. Em ambos os casos, observou-se que a gata permaneceu perdida, relutante em andar e se chocando contra alguns objetos. Sempre que andava, a paciente utilizava suas vibrissas para sentir os obstáculos à sua frente. Como exame complementar, tiraram-se fotos das retinas com o aparelho Clear View $\AA$. Por meio destas, visualizou-se, bilateralmente, ausência de vasos, focos de hiperrefletividade na área tapetal e despigmentação da área não-tapetal (Figura 4). 
Figura 4 - Fotos das retinas da gata, 11 anos, SRD, em agosto de 2014. Notar a ausência de vascularização e hiperrefletividade tapetal em ambos os olhos. A: olho direito. B: olho esquerdo. Fotos tiradas com 0 aparelho ClearView®.

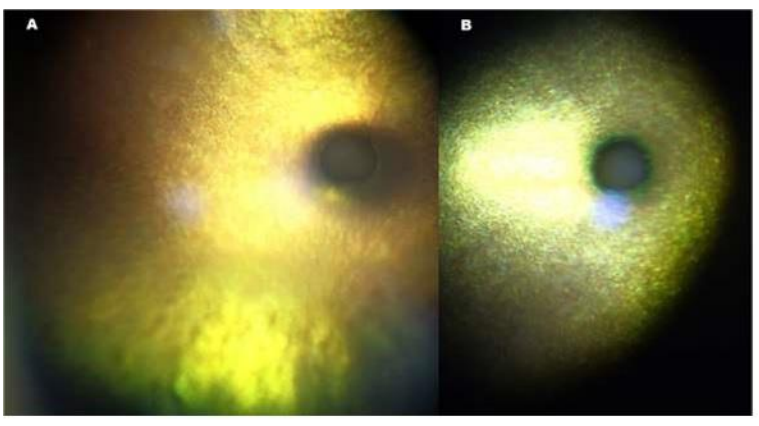

Além das fotos, realizou-se a eletrorretinografia de ambos os olhos. Por meio desta, verificou-se ausência total de resposta da retina, ou seja, disfunção de bastonetes e cones. Considerando o histórico de dificuldade progressiva de enxergar, adicionado ao exame clínico e à avaliação da retina por meio do ERG, conclui-se que a paciente apresentava Atrofia Progressiva de Retina. Por ser uma doença hereditária e degenerativa, já em estágio avançado, não há tratamento específico para este caso. Indicam-se apenas avaliações periódicas para monitoramento da progressão da doença.

\section{RESULTADOS E DISCUSSÃO}

O caso relatado trata de uma gata, com 11 anos de idade, SRD, com ocorrência de cegueira progressiva na fase senil. Dentre as possíveis causas de cegueira em gatos, pode-se citar, além da Atrofia Progressiva de Retina, o Glaucoma, Descolamento da Retina, Panuveíte, Isquemia Retiniana secundária à hipertensão arterial sistêmica, deficiência de Taurina, Retinopatia Tóxica, causada pela alta dose de enrofloxacina, ou até mesmo uma Cegueira de caráter central.
De acordo com a anamnese e o exame clínico do caso descrito, não foram observadas, além da retina, outras alterações em todo o bulbo ocular, exceto pela midríase e pela lesão tipo Florida spots. Sendo assim, doenças e alterações como o glaucoma, descolamento de retina, panuveíte ou isquemia retiniana são previamente excluídas. Em todos esses casos observa-se, geralmente, uma cegueira súbita, sendo algumas irreversíveis, como o glaucoma e o descolamento de retina.

A APR, caracterizada por ser uma degeneração retiniana hereditária (PETERSEN-JONES, 2005), pode ser classificada como distrofia ou degeneração da retina (PETERSENJONES, 1998). Na distrofia, não há a formação normal dos fotorreceptores durante a maturação da retina, enquanto que na degeneração observa-se a morte dos fotorreceptores após a maturação da mesma. Portanto, a degeneração ocorre mais tardiamente, sendo que a cegueira completa em geral é observada após sete anos de idade (GRAHN \& SANDMEYER, 2009). Em ambos os casos são observados, inicialmente, nictalopia seguida de acromatopsia, de acordo com a evolução da doença (NARFSTRÖM et al.,2011). Animais acometidos apresentam hiperrefletividade simétrica e bilateral da região tapetal da retina, além de atenuação dos vasos retininanos. Para confirmar o diagnóstico, realiza-se a eletrorretinografia (SANDE, 2010). Uma vez que os sinais retinianos da APR são semelhantes aos apresentados pela paciente e, também, a evolução da doença e os sinais clínicos observados pelos proprietários ao decorrer do tempo correspondem com degeneração da retina, suspeitou-se de APR. Logo, foi realizada a eletroretinografia, por meio da qual se determinou a morte celular da retina. Ainda em relação aos diagnósticos diferencias para a APR, a 
deficiência de Taurina é caracterizada pela apresentação de degeneração retiniana com ocorrência de cegueira completa do animal após um ano com dietas deficientes do aminoácido (Stades et al.,1999). De acordo com Slatter (2005), observam-se lesões hiperrefletivas focais na retina, as quais se tornam difusas com o tempo. Além disso, o animal com deficiência de taurina pode apresentar falhas reprodutivas e cardiomiopatia dilatada (NOVOTNY et al.,1994). No caso relatado, a gata era alimentada com ração para gatos da marca Hills $\AA$ desde filhote, ração esta que possui formulação adequada com $1000 \mathrm{mg} / \mathrm{kg}$ de taurina (Hill's Pet Nutrition, Inc, 2014).

A Retinopatia Tóxica, caracterizada pelo uso indiscriminado de enrofloxacina em gatos em tratamentos prolongados com doses elevadas (WIEBE \& HAMILTON, 2002), promove sinais oftálmicos de intoxicação, como midríase, ausência de reflexos pupilares e colisão em objetos (GELATT et al.,2007), além de hiperrefletividade difusa e bilateral da zona tapetal e atenuação dos vasos retinianos (GELATT et al.,2007; FORD et al.,2007). Além disso, podem-se observar sinais sistêmicos de intoxicação como emese, sialorréia, piloereção, mobilidade reduzida e taquipnéia (MESSIAS, 2008). O histórico da paciente em questão refere a ocorrência de um acidente com um ano de idade, o que a manteve internada durante um mês. Considerando a possibilidade de ter recebido neste período a administração de enrofloxacina devido ao seu grande espectro de ação, a retinopatia tóxica seria o principal diagnóstico a se considerar caso os sinais de cegueira ocorressem na época discorrida. Como o déficit visual apareceu 10 anos após o evento da internação e de maneira progressiva, não é possível dizer, com certeza, que a cegueira seja decorrente de uma possível administração inadequada de enrofloxacina há 10 anos, já que esta retinopatia é caracterizada por uma cegueira súbita (GELATT, 2007). A excreção da enrofloxacina pelo organismo é realizada rapidamente e em altas concentrações pela urina, sendo esta impossibilitada somente em casos de falência renal (VANCUTSEM, BABISH \& SCHWARK, 1990), o que não ocorreu com a paciente.

Em caso de confirmação de APR, não há tratamento que possa reverter os danos causados em um animal acometido pela doença. Ainda assim, estudos recentes acreditam que a terapia com suplemento vitamínico possa prevenir e até mesmo retardar a progressão da degeneração retiniana. Acredita-se que o suplemento vitamínico diminua a incidência do estresse oxidativo fisiológico, causado pelo envelhecimento, pela exposição à luz ultravioleta e pelo próprio metabolismo celular, além de retardar o estresse oxidativo causado pela APR (GELATT et al.,2007). Porém, por ser uma doença progressiva e degenerativa, quando são diagnosticadas já em estágio avançado, não há tratamento possível, sendo necessário apenas realizar 0 acompanhamento da evolução da doença.

Em gatos, quando há partes danificadas da retina, independente de qual seja a causa, os neurônios no córtex visual associados a essas partes da retina se tornam, subsequentemente, associados a regiões da retina com funcionamento normal. Isso torna difícil a estimação da função visual após o dano retiniano e contribui para a visão aparentemente superior ao que seria esperado em alguns pacientes com lesões retinianas extensivas (SLATTER, 2005). No caso relatado, foi realizado durante a consulta o teste de obstáculos, com a iluminação da sala presente e ausente. Em ambos os casos, a paciente permaneceu perdida e insegura, principalmente por estar em um 
ambiente estranho, porém pareceu de certa forma adaptada à sua deficiência visual, utilizando com frequência suas vibrissas para sentir os obstáculos à sua frente. Acredita-se, por isso, que ela já se encontrava em um estágio avançado e adaptado de cegueira, podendo estar se adaptando há muitos anos, inclusive sem a percepção por parte dos proprietários até um determinado momento.

\section{CONCLUSÃO}

Embora a doença já tenha sido ricamente descrita na literatura, o caso relatado se torna atípico por se tratar de uma gata sem raça definida, já que a Atrofia Progressiva de Retina é uma doença que, quando acomete gatos, fato este mais raro que em cães, geralmente acomete a raça Abssínio, Somali, Siamês e Persa.

Apesar de o histórico da paciente não condizer com as características de uma retinopatia tóxica, não há como excluir completamente essa possibilidade caso a droga tenha sido realmente administrada, pois nos estágios finais da APR as alterações retinianas são semelhantes às de intoxicação por enrofloxacina e, além disso, não há comprovação genética de que a paciente é portadora do alelo responsável pela APR. Para a confirmação definitiva do diagnóstico, seria necessário realizar um teste genético hoje em dia oferecido comercialmente, tal como o da empresa inglesa LABOKLIN®.

\section{REFERÊNCIAS}

BEDFORD, P. G. C. Progressive retinal atrophy in dogs. Veterinary Record., v.4, n.15, p.124, 2005.

BARNETT K. C, CURTIS R. Autosomal dominant progressive retinal atrophy in
Abyssinian cats. Jornal of Heredity, v.76, n.3, p.168-170, 1985.

CURTIS R, BARNETT K. C, LEON A. An Early-Onset Retinal Dystrophy With Dominant Inheritance in the Abyssinian cat. Clinical and Pathological Findings. Investigative Ophthalmology e Visual Science, v.28, n.1, p. 131-139, 1987.

FORD, M. M. et al.Ocular and systemic manifestations after oral administration of a high dose of enrofloxacin in cats. American Journal of Veterinary Research, v.2, n.68, p.190-202, 2007.

GELATT, K. N. Veterinary Ophthalmology. Blackwell Publishing Ltda, 2007, p.690-1274.

GELATT K. N. Manual de Oftalmologia Veterinária. Manole, 2003, p.227-252.

GIULIANO, E. A \& VAN DER WOERDT, A. Feline retinal degeneration: Clinical experience and new findings (19941997). Journal of the American Animal Hospital Association, v.35, n.6, p.511514, 1999.

GLAZE, M. B. Congenital and hereditary ocular abnormalities in cats. Clinical Techniques in Small Animal Practice, v.2, n.20, p.74-82, 2005.

GOMES, D. et al.Atrofia progressiva generalizada da retina em cães da raça Cocker Spaniel. Ciência Rural, v.43, n.8, p.1405-1414, 2013.

GRAHN, B. H; SANDMEYER, L. S. Diagnostic Ophthalmology. Progressive Retinal Atrophy. The Canadian Veterinary Journal, v.7, n. 50, p. 775-776, 2009.

GROZDANIC, S. D. et al.. Differential diagnosis of retina and optic nerve diseases in dogs by pupil light reflex analysis: functional implications for SARD diagnosis. In: $37^{\circ}$ ACVO, p.9, 2006.

HERRERA, D. Oftalmología clínica en animales de compañia. Buenos Aires: Inter-Médica, 2007. p.73-83. 
HILL'S PET NUTRITION, 2014, Disponível em: http://www.hillspet.com.br. Acesso em: 19 de novembro de 2014.

MENOTTI-RAYMOND M. et al. Widespread retinal degenerative disease mutation ( $\mathrm{rdAc}$ ) discovered amonga large number of popular cat breeds. The Veterinary Journal, v.186, n.1, p.32-38, 2010.

MENOTTI-RAYMOND M. et al. Mutation in CEP290 discovered for cat model of human retinal degeneration. Jornal of Heredity, v.98, n.3, p.211-220, 2007.

MESSIAS, A. et al. Retinal safety of a new fluorquinolone, pradofloxacin, in cats: assessment with eletroretinography. Documenta Ophthalmologica, v.116, n.3, p.177-191, 2008.

NARFSTRÖM, K. et al. Characterization of feline hereditary retinal dystrophies using clinical, functional, structural and molecular genetic studies. Veterinary Ophthalmology, v.2011, n.1, p.30-36, 2011.

NARFSTRÖM, K. \& EKESTEN, B. Electroretinographic evaluation of Papillons with and without hereditary retinal degeneration. American Journal of Veterinary Research, v.59, n.2, p.221226, 1998.

NARFSTRÖM, K.; WILÉN, M.; ANDERSSON, B. E. Hereditary retinal degeneration in the Abyssinian cat: Developmental studies using clinical electroretinography.

Documenta Ophthalmologica, v.69, n.2, p.111-118, 1988.

NARFSTRÖM, K. Hereditary progressive retinal atrophy in the Abyssinian cat. Journal of Heredity, v.74, n.4, p. 273276, 1983.

NOVOTNY, M. J.; HOGAN, P. M.; FLANNIGAN, G. Echocardiographic evidence for myocardial failure induced by taurine deficiency in domestic cats.
Canadian Journal of Veterinary Research, v.58, n1, p.6-12, 1994.

PETERSEN-JONES, S. M. A review of research to elucidate the causes of the generalized progressive retinal atrophies. Veterinary Journal, v. 155, n.1, p.5-18, 1998.

PETERSEN-JONES S.M. Animal models of human retinal dystrophies. Eye (London), v.12, p. 566-570, 1998.

PETERSEN-JONES, S. M. Advances in the molecular understanding of canine retinal diseases. Journal of Small Animal Practice, v.8, n.46, p.371-380, 2005.

$\mathrm{RAH}, \mathrm{H}$. et al. Early-onset, autosomal recessive, progressive retinal atrophy in Persian cats. Investigative Ophthalmology \& Visual Science, v.5, n.46, p.1742-7, 2005.

RUBIN, L.F. \& LIPTON, D.E. Retinal degeneration in kittens. Journal of the American Veterinary Medical Association, v.162, n.6, p.467-469, 1973. SAFATLE, A. M. V. et al. Determinação dos valores normais do eletrorretinograma de campo total em cães da raça Poodle portadores de catarata de acordo com a faixa etária. Ciência Rural, v.40, n.3, p.587-593, 2010.

SANDE, P. Enfermidade de la Retina Felina. X Congresso Nacional de Aveaca, 2010. Disponível em: http://aveaca.org.ar/Proceeding\%20AVE ACA.pdf\#page $=41$. Acesso em: 10 de setembro de 2014.

SLATTER, D. 2005. Fundamentos de Oftalmologia Veterinária. São Paulo: Roca, 2005.

STADES, F.C. et al. Fundamentos de Oftalmologia Veterinária. São Paulo: Manole, 1999.

VANCUTSEM, P.M.; BABISH, J.G.; SCHWARK, W.S. The fluorquinolone antimicrobials: structure, antimicrobial activity, pharmacokinetics, clinical use in 
domestic animals and toxicity. The Cornell Veterinarian, v.80, n.2, p.173186, 1990.

WIEBE, V. \& HAMILTON, P. Fluorquinolone-induce retinal degeneration in cats. Journal of the American Veterinary Medical Association. v. 221, n.11, p.1568-1571, 2002. 